\title{
Modified supine position for the management of malignant ureteral obstruction
}

\begin{abstract}
Introduction: The treatment of ureteral obstruction due to advanced malignancy is a therapeutic challenge. We propose a new position that allows simultaneous transurethral and transrenal access using a combined approach with eventual endoscopic resection.

Material and methods: 24 patients (44 ureters) underwent ureteral repermeabilisation for malignant ureteral obstruction in the modified supine position. The flank is elevated 30 to $45^{\circ}$ with regard to horizontal plane and lower limbs are split and bent in a lower position. After a trial of retrograde catheterization, the kidney is punctured. A straight hydrophilic guide wire and a special intrarenal manipulation catheter were used to cross the malignant ureteric stricture. If not a bladder resection was performed in the same position in direction of the wire with fluoroscopic guidance. The guide wire was then advanced into the bladder. The double-pigtail stent was then placed.
\end{abstract}

Results: Mean age was 64.2 years (32-69). Repermeabilisation were possible in $41(93.1 \%)$ of 44 ureters attempted by the procedure. Trigonal resection was necessary in 12 patients. Median length of stenosis was $2.6 \mathrm{~cm}(1.6-5 \mathrm{~cm})$. No abdominal or thoracic organ injuries were reported. Mean operating room time was $53 \mathrm{~min}$ (25-120min). Mean hospital stay was three days (2-6days).After repermeabilisation, serum creatinine levels decreased significantly from a mean $127.3 \mathrm{mg} / \mathrm{l}$ (range, $18-706 \mathrm{mg} / \mathrm{l}$ ) to $16.2 \mathrm{mg} / \mathrm{l}$ (range, $5-28 \mathrm{mg} / \mathrm{l}$; $\mathrm{p}<0.0001)$.

Conclusion: The modified supine position an important alternative to nephrostomy. It might also be useful for the treatment of other pathologies essentially urolithiasis.

Keywords: ureteral obstruction, neoplasms, disease management, stents, nephrostomy, percutaneous
Volume 5 Issue 3 - 2017

\author{
Youness El Harrech, Ghoundale O, Zaini R \\ and Touiti D \\ Department of urology, Military hospital Avicenne, Morocco
}

Correspondence: Youness El Harrech, Department of urology, service d'urologie, Hôpital Militaire Avicenne, Marrakech, Marocco, Tel 00 212661326160,

Email youness.elharrech@gmail.com

Received: July 29, 2017| Published: September 20, 2017

\section{Introduction}

The treatment of ureteral obstruction due to advanced abdominal or pelvic malignancy is a therapeutic challenge. This type of obstruction is usually due to metastatic disease by direct tumor compression of the ureters (cervical bladder, prostate or colorectal), extrinsic compression from expansion of the retroperitoneal cavity (retroperitoneal soft tissue or sarcoma) or encasement of the ureters with metastatic lymph nodes. ${ }^{1}$ The optimal management of such obstruction resulting from advanced malignancy remains unclear. ${ }^{2}$ The finding of ureteral obstruction due to malignancy is an ominous sign with a resulting median survival of 3 to 7 months. ${ }^{3}$ Progressive obstructive uropathy may likely lead to clinical manifestations, such as uremia, electrolyte imbalances and persistent urinary tract infections, if obstruction is not bypassed. ${ }^{4}$ Percutaneous nephrostomy has been traditionally used to provide temporary or long-term urinary drainage for patients with impassable ureteric strictures. This procedure has major disadvantages for long-term use because there is a need for regular changes, the catheters often get dislodged and/or blocked, and the external drainage with catheter and bag often contributes to an impaired quality of life for the patients. ${ }^{5}$ Managing extrinsic compression of the ureter by retrograde stenting is first-line therapy but involves a guarded decision because stent failure occurs in nearly half of the treated patients. ${ }^{6,7}$ Because this high level of failure and the high rate of complications of permanent nephrostomy, our study proposes a novel position that allows easier stenting via combined and simultaneous antegrade and retrograde access of the obstruction.

\section{Materials and methods}

\section{Patients}

A retrospective study was performed on 24 consecutive patients receiving an in dwelling ureteral stent using the presented technique. Preoperative evaluation of the patients included history, clinical examination, routine laboratory investigations (basal parameters including negative urine cultures), radiological investigations (plain abdominal x-ray, ultrasonography, and non-contrast and contrast computerized tomography CT scan), and evaluation of the anaesthesiological risk according to the American Society of Anesthesiologists (ASA) classification of physical status. ${ }^{8}$

\section{Surgical technique}

All procedures were carried out under general anesthesia. The patient was placed in the modified supine position. The flank is elevated 30 to $45^{\circ}$ with regard to horizontal plane and lower limbs are split and bent in a lower position (Figure 1). The retrograde approach was used initially in all cases. If unsuccessful (Failure to find ureteric orifice or Failure to cross stricture), antegrade or both antegrade and retrograde approach was attempted. The most accessible posterior calyx was punctured under ultrasound guidance using a $22 \mathrm{G}$ Chiba needle and the tract dilated to $10 \mathrm{Fr}$. Radiopaque contrast and Methylene blue were injected into the renal cavity. The renal pelvis, Pelviureteric junction and calyces were identified as the seat and length of the stricture (Figures $2 \mathrm{~A} \& 2 \mathrm{~B}$ ). Once the pelviureteric junction 
was crossed and the ureter accessed, a straight hydrophilic guide wire and a special intrarenal manipulation catheter were used to cross the malignant ureteric stricture (Figure 3). If the stenosis was too tight and that the guide failed to pass, a resection guided with fluoroscopy in the direction of the guide was performed in the same flank modified position. The resection was continued until visualization of the guide and Methylene blue.

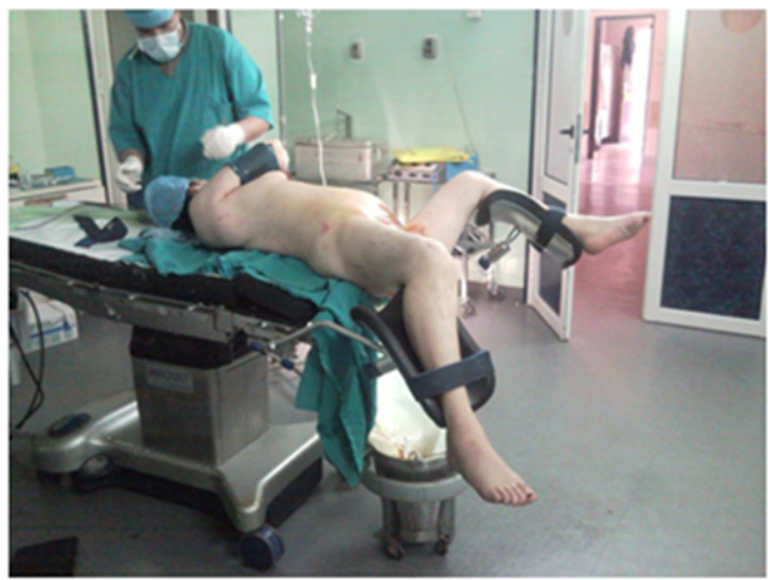

Figure I Modified supine position:The flank is elevated 30 to $45^{\circ}$ with regard to horizontal plane and lower limbs are split and bent in a lower position.

The wire was then advanced into the bladder and preferably harbored with an extracting forceps. This guide wire was then exchanged for an ultra-stiff guide wire. The end of the guide-wire is secured with a clamp at the skin level and the desired steerable stent is positioned under radiographic control in a retrograde fashion over the harbored tip of the guide-wire. Finally, the wire is extracted through the puncture and the stent is released in its correct position. In some cases, dilatation of the ureteric stricture was performed. We used for this purpose an Amplatz sheath, through which progressive increasing size ureteral stents were passed. Applying some tension on the extremities of the guide wire facilitates this maneuver (Figure 4). A final fluoroscopic image was stored to document the stent position. An 8 Frnephrostomy drainage catheter was placed and removed in 48-hours following a satisfactory nephrostogram.

\section{Results}

Over a 2 -year study period 24 patients ( 17 male, 7 female) with malignant ureteric obstruction were included in the study. Mean age at the time of implantation of ureteral stent was 64.2 years (range, 32 69). From the study group assembled 11 patients had prostate cancer, four patients had colonic cancer, four had bladder cancer, and five had cervical cancer. All patients had a unilateral or bilateral dilated upper urinary tract and elevated creatinine levels. Four patients were admitted for anuria and had initial PCN. Median length of stenosis was $2.6 \mathrm{~cm}$ (range $1.6-5 \mathrm{~cm}$ ). Repermeabilisation were possible in 41 $(93.1 \%)$ of 44 ureters attempted by the procedure. Trigonal resection was necessary in 12 patients. There were three failures, which were due to inability to cross the lower third malignant lesion. 11 patients had minor urinary hemorrhage following the resection or nephrostomy, however, only one patient required blood transfusion. Two patients presented transient fevers during the first two postoperative days that were promptly responsive to specific broad-spectrum antibiotic therapy. No abdominal, vascular or thoracic organ injuries were reported. Mean operating room time for each ureter was $53 \mathrm{~min}$ (range, 25-120min). After repermeabilisation, hydronephrosis was completely resolved in 37 kidneys and reduced in the remaining 4 . Accordingly, serum creatinine levels decreased significantly from a mean $127.3 \mathrm{mg} / \mathrm{l}$ (range, $18-706 \mathrm{mg} / \mathrm{l}$ ) to $16.2 \mathrm{mg} / 1$ (range, $5-28 \mathrm{mg} / 1$; $<0.0001$ ). Mean hospital stay was three days (range, 2-6 days).

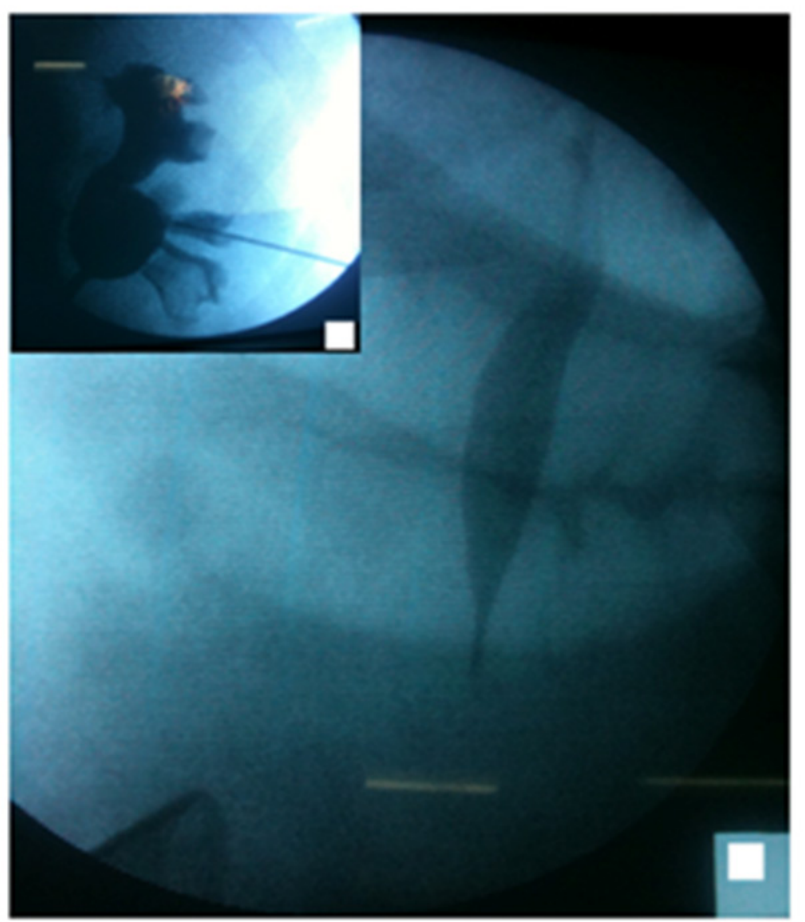

Figure 2

A: Identification of the renal pelvis, pyeloureteral junction and calyces and kidney access.

B: Identification of the stenosis and its length.

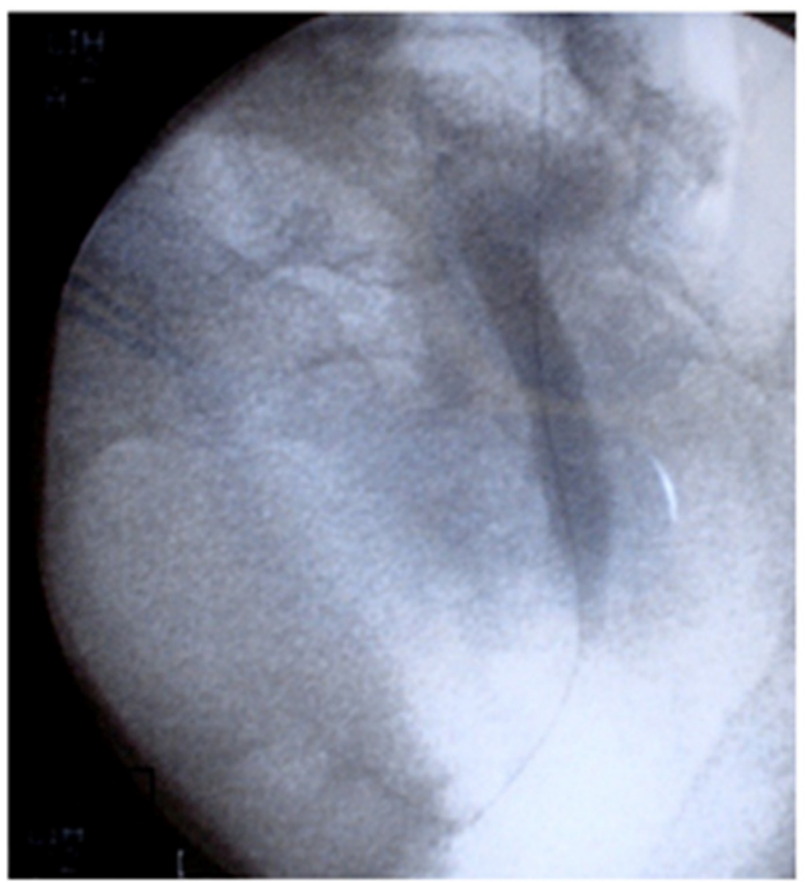

Figure 3 A straight hydrophilic guide wire and a special intra-renal manipulation catheter were used to cross the malignant ureteric stricture. 


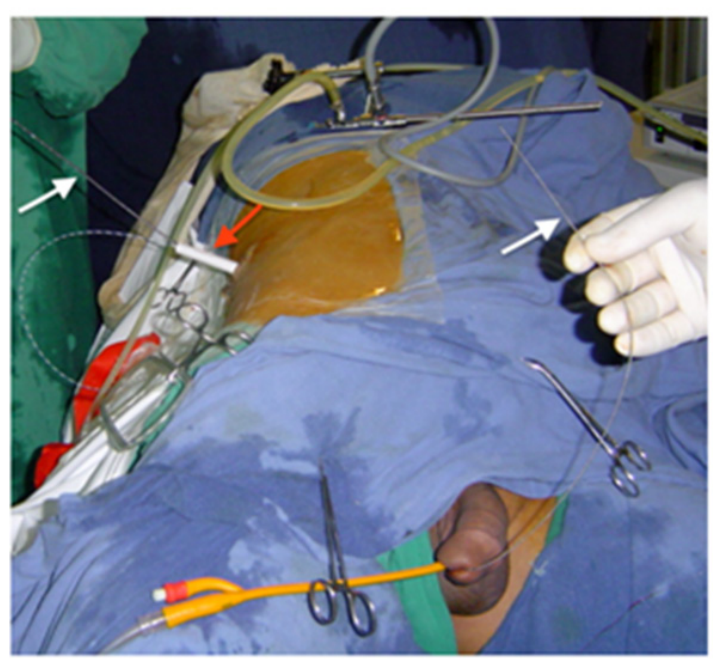

Figure 4 Dilatation of the ureteric stricture through an Amplatz Sheath (red arrow): note the guide wire is introduced though the renal access and extracted through the urethral meatus (white arrows).

\section{Discussion}

The management of extrinsic malignant ureteral obstruction is a difficult situation in which the urologist balances patient quality of life, renal preservation and risk of complication in the setting of a poor prognosis. Although there are many options, there is a lack of consensus on the optimal management of extrinsic malignant ureteral obstruction. Still, urinary diversion may be appropriate in certain subgroups with advanced malignancy. It has been shown to allow patients to be removed from hemodialysis due to acute pulmonary edema, hyperkalemia or refractory metabolic acidosis resulting from renal obstruction. ${ }^{4}$ Additionally, patients with symptomatic ureteral obstruction may be relieved of intractable flank pain after decompression by PCN. ${ }^{9}$ Retrograde insertion of ureteral stents ultimately fails in $16 \%$ to $58 \%$ of patients with obstruction due to malignancy. These patients then ultimately require a PCN or antegrade stent to decompress the urinary tract. ${ }^{2}$ However, a success rate of retrograde stenting of $75 \%$ to $84 \%$ has been reported in several studies. ${ }^{2}$ The difference in success rates may be related to the type of pelvic malignancy. For example, ureters obstructed by bladder, prostatic or cervical cancer have been found to be managed in retrograde fashion with a success rate of only $15 \%$ to $21 \% .^{10}$

Interestingly ureteral invasion of malignancy at cystoscopic examination is highly predictive of eventual retrograde stent failure. With our technique and this new position, ureteral stenting was successful in $(93.1 \%)$ of case, even if most of malignancy were prostate cancer, bladder and cervical cancer. The fear of colonic or splanchnic organ injuries has probably conditioned patients' prone positioning when the technique of percutaneous nephrostomy was first described. ${ }^{11}$ The prone position may, however, be contraindicated for anesthesiologic reasons because of circulatory and ventilatory difficulties, especially in obese patients. ${ }^{12,13}$ Moreover, it can be difficult in patients with limb contracture. Modified positions were suggested to overcome such problems. Valdivia Uria et al.. ${ }^{4}$ proposed PCNL in a completely supine position with a 3-1 water bag below the ipsilateral flank and with the ipsilateral leg totally extended. Performing renal accesses inhuman corpses, they demonstrated no damage tothe peritoneum and colon, and with a computed tomography study, they confirmed that in the supine position, the colon was more distant from the kidney than in the prone position. Starting in 2004, we routinely adopted the modified supine position to perform PCNL in patients affected by large and/or complex urolithiasis. We decide to use the same position since 2007 in percutaneous repermeabilisation for neoplastic ureteral stenosis due to pelvic malignancy.

Anaesthesiological advantages include absence of cardiovascular, respiratory, neuroendocrine, and pharmacokinetic problems typical of the prone position, particularly in obese patients. ${ }^{15}$ Urological advantages include easier puncture of the kidney, creation of a 2-tiered field to respect aseptic conditions and allowed simultaneous antegrade and retrograde endoscopic approach to urinary tract. (Resection was made in 12 patients in our study). Management advantages include: ease of patient positioning, which is not particularly laborious or timeconsuming; more patient comfort, which might even enable the use of less intense anesthesia; ${ }^{16}$ less manipulation of the anesthetized patient and no need for a position change from the lithotomy to the prone position during the procedure and then from prone to the lithotomy position to grasp the wire, knowing that this repositioning is more demanding for the surgical team and include occupational risk due to shifting of heavy loads, especially in case of obese patients; less risk of pressure injuries related to inaccurate repositioning, which are possibly responsible for ligament lesions, visual problems, and neurological deficits (including brachial plexus palsy and myelic lesions causing paraplegia); $\mathrm{x}$-ray exposure is reduced because puncture and dilation of the nephrostomy tract are quite perpendicular to the body and the operator's hands are outside the fluoroscopic field; ${ }^{15}$ and operating room time is decreased.

Positions similar to ours were reported in the literature. Galdakaomodified supine Valdivia (GMSV) position allows simultaneous performance of PCNL and retrograde ureteroscopy (ECIRS, Endoscopic Combined Intra-Renal Surgery). ${ }^{17}$ Advantage of our position is that lowering the patient legs facilitates caliceal puncture and nephrostomy tract dilation, augments the distance from the lower rib to the iliac crest. Additionally, stretching the tissues in this area of the abdominal wall avoids redundancy or folding of the tissue layers and further helps develop percutaneous access. Our position might also be useful for the treatment of other pathologies, including urolithiasis, realignment of ureteral stumps; uretero-colic and ureteroileal anastomotic strictures in urinary diversions; reno-ureteral pathologies in transplanted.

Alternative urinary diversion includes percutaneous nephrostomy $(\mathrm{PCN})$. Open nephrostomy tube placement in patients with ureteral obstruction with advanced malignancy is historically associated with a $45 \%$ major complication rate and a $25 \% 30$-day mortality rate. ${ }^{1} \mathrm{~A}$ recent study also showed a major complication rate of $53 \%$, which reflects the gravity of disease despite advancement in insertion technique..$^{18}$ One study demonstrated that only 11 of 17 patients with a PCN had acceptable QOL for 2 months or more. ${ }^{19}$ Concurrently study results have shown a high incidence of complications associated with long-term management of PCN tubes. One study showed a $10 \%$ dislodgment rate of PCN tubes, requiring replacement. This same study also demonstrated a $65 \%$ rate of febrile urinary tract infections. These febrile urinary tract infections can certainly have a deleterious impact on patient QOL. ${ }^{20}$ Other problems that can contribute to poor QOL after PCN placement are urinary leakage and skin excoriation at the nephrostomy exit site..$^{21}$ Then most of authors, recommend a trial of retrograde ureteral stents in all patients. In case of failure, $\mathrm{PCN}$ is performed. ${ }^{10}$

Another alternative is a subcutaneous nephro-vesical bypass procedure, which eliminates the presence of PCN tubes. This 
minimally invasive procedure is performed using general anesthesia. A composite silicones heath is introduced into the kidney and advanced subcutaneously to the bladder under fluoroscopic guidance. The tube is then brought into the bladder and secured by sutures placed cystoscopically. Investigators have found no dislodgment or encrustation of the diversion tube. Moreover, the investigators found a significant improvement in patient QOL, as measured by the validated European Organization for the Research and Treatment of Cancer QLQ-C30 scale, After PCN removal and nephro-vesical bypass placement up to 18 months after the procedure. Patients were able to void normally, which can also have significant impact on QOL compared to that with indwelling Foley catheters or wearing diapers at the end of life..$^{22}$ In another study, in five patients (17.9\%) of 31 , the system had to be replaced due to occlusion at a mean follow-up of 10.2 months. ${ }^{23}$

More investigations are needed of this method before it should be routinely used. Moreover, this procedure is proposed as a final solution for patients with advanced malignant disease with limited life expectancy, where internal ureteral stenting proved to be impossible. The Detour stent costs approximately 2200 Euro and usually involves a 3-d hospital stay. Our technique is proposed for patients with locally involvement not in the final step of their life and proved that internal stenting is most often possible. Moreover, it is less expensive. Weaknesses of our series include the small population size. Although this is the first series to date in the use of such us position in the management of malignant ureteral compression. Another weakness is the lack of validated quality of life measures. A future prospective study could enroll a larger number of patients, and compare the progression, complications and quality of life between percutaneous nephrostomy tubes and the technique reported.

\section{Conclusion}

The high rate of failure of standard retrograde ureteral stenting, the high complication rate of nephrostomy and frequent rehospitalisation render the repermeabilisation an important alternative for urinary diversion in patient with ureteral obstruction in advanced malignancies. The modified supine position resulted in decreased operating room time, less manipulation of the anesthetized patient, creation of a 2-tiered field to respect aseptic conditions, and allowed simultaneous antegrade and retrograde endoscopic approach to upper urinary tract. Given the overall prognosis, risk of complications and internal stent failures, an open discussion with the patient and family should be pursued to determine the best treatment option.

\section{Acknowledgements}

None.

\section{Conflicts of interest}

All authors declared there are no conflicts of interest.

\section{Funding}

None.

\section{References}

1. Russo P. Ureteral obstruction and stents:still a difficult problem for patients and urologists alike. J Urol. 2005;174(6):2088.

2. Kouba E, Wallen EM, Pruthi RS. Management of Ureteral Obstruction Due to Advanced Malignancy: Optimizing Therapeutic and Palliative Outcomes. J Urol. 2008;180(2):444-450.

3. Russo P. Urologic emergencies in the cancer patient. Semin Oncol. 2000;27(3):284-298.
4. Romero FR, Broglio M, Pires SR, et al. Indications for percutaneous nephrostomy in patients with obstructive uropathy due to malignant urogenital neoplasias. Int Braz J Urol. 2005;31(2):117-124.

5. Hepperlen TW, Mardis HK, Kammandel H. The pigtail ureteral stent in the cancer patient. J Urol. 1979;121(1):17-18.

6. Chung SY, Stein RJ, Landsittel D, et al. 15-year experience with the management of extrinsic ureteral obstruction with indwelling ureteral stents. J Urol. 2004;172(2):592-595.

7. Yossepowitch O, Lifshitz DA, Dekel Y, ross M, Keidar DM, et al. Predicting the success of retrograde stenting for managing ureteral obstruction. J Urol. 2001;166(5):1746-1749.

8. Wolters U, Wolf $\mathrm{T}$, Stutzer $\mathrm{H}$, et al. ASA classification and perioperative variables as predictors of postoperative outcome. Br J Anaesth. 1996;77(2):217-222.

9. Shekarriz B, Shekarriz H, Upadhyay J, et al. Outcome of palliative urinary diversion in the treatment of advanced malignancies. Cancer. 1999;85(4):998-1003

10. Ganatra AM, Loughlin KR. The management of malignant ureteral obstruction treated with ureteral stents. J Urol. 2005;174(6):2125-2128.

11. Goodwin WE, Casey WC, Woolf W. Percutaneous trocar (needle) nephrostomy in hydronephrosis. J Am Med Assoc. 1995;157(11):891894

12. Peces-Barba G, Rodriguez-Nieto MJ, Verbanck S, et al. Lower pulmonary diffusing capacity in the prone vs. supine posture. $J$ Appl Physiol. 2004;96(5):1937-1942.

13. Pump B, Talleruphuus U, Christensen NJ, et al. Effects of supine, prone, and lateral positions on cardiovascular and renal variables in humans. Am J Physiol Regul Integr Comp Physiol. 2002;283(1):R174-R180.

14. Valdivia Uria JG, Valle Gerhold J, Lopez Lopez JA, et al. Technique and complications of percutaneous nephroscopy:experience with 557 patients in the supine position. J Urol. 1998;160(6 Pt 1):1975-1978.

15. Ibarluzea G, Scoffone CM, Cracco CM, et al. Supine Valdivia and modified lithotomy position for simultaneous anterograde and retrograde endourological access. BJU Int. 2007;100(1):233-236.

16. Aravantinos E, Karatzas A, Gravas S, et al. Feasibility of percutaneous nephrolithotomy under assisted local anaesthesia:a prospective study on selected patients with upper urinary tract obstruction. Eur Urol. 2007;51(1):224-228.

17. Scoffone CM, Cracco CM, Cossu M, et al. Endoscopic combined intrarenal surgery in Galdakao-modified supine Valdivia position:a new standard for percutaneous nephrolithotomy? Eur Urol. 2008;54(6):13931403.

18. Wong LM, Cleeve LK, Milner AD, et al. Malignant ureteral obstruction:outcomes after intervention. Have things changed? J Urol. 2007;178(1):178-183.

19. Emmert C, Rassler J, Kohler U. Survival and quality of life after percutaneous nephrostomy for malignant ureteric obstruction in patients with terminal cervical cancer. Arch Gynecol Obstet. 1997;259(3):147151

20. $\mathrm{Ku} \mathrm{JH}$, Lee SW, Jeon HG, et al. Percutaneous nephrostomy versus indwelling ureteral stents in the management of extrinsic uretera obstruction in advanced malignancies:are there differences? Urology. 2004;64(5):895-899.

21. Schmidbauer J, Kratzik C, Klingler HC, et al. Nephrovesical subcutaneous ureteric bypass:long-term results in patients with advanced metastatic disease-improvement of renal function and quality of life. Eur Urol. 2006;50(5):1073-1078.

22. Desgrandchamps F, Leroux S, Ravery V, et al. Subcutaneous pyelovesical bypass as replacement for standard percutaneous nephrostomy for palliative urinary diversion:prospective evaluation of patient's quality of life. J Endourol. 2007;21(2):173-176.

23. Schmidbauer J, Kratzik C, Klingler HC, et al. Nephrovesical subcutaneous ureteric bypass:long-term results in patients with advanced metastatic disease-improvement of renal function and quality of life. Eur Urol. 2006;50(5):1073-1078. 